\title{
Excitation Density Dependence of Optical Oxygen Sensing in Poly(9,9-dioctylfluorene) Waveguides Showing Amplified Spontaneous Emission
}

\author{
M. Anni and S. Lattante \\ Dipartimento di Matematica e Fisica "Ennio De Giorgi," Università del Salento, Via per Arnesano, 73100 Lecce, Italy \\ Correspondence should be addressed to M. Anni; marco.anni@unisalento.it
}

Received 10 January 2014; Accepted 3 February 2014; Published 4 March 2014

Academic Editors: P. Bhowmik and E. J. Nassar

Copyright ( $\odot 2014$ M. Anni and S. Lattante. This is an open access article distributed under the Creative Commons Attribution License, which permits unrestricted use, distribution, and reproduction in any medium, provided the original work is properly cited.

Reversible oxygen induced emission quenching of both the Spontaneous Emission (SE) and the Amplified Spontaneous Emission (ASE) of poly(9,9-dioctylfluorene) waveguides is demonstrated. We show that ASE shows a stronger quenching than SE, up to about 6.2 times, but also a stronger decrease when the excitation density increases. We conclude that the fast increase of the ASE decay rate is the main process in determining the ASE detection sensitivity, limiting the potentiality of sensitivity improvement of ASE with respect to SE.

\section{Introduction}

The development of novel active systems for gas sensing is receiving increasing attention due to the very wide range of possible applications. In particular the detection of oxygen is particularly interesting in the medical field for the monitoring of oxygen content in both air and blood, as well as in the environmental monitoring field [1]. Among the different proposed techniques, exploiting the oxygen effects on the electric conductivity [2], material color [3], and chemiluminescence [4], optical sensors, based on the oxygen induced photoluminescence quenching, are characterized by fast response time and high sensitivity. The typical active systems are blends between an inert matrix and a phosphorescent molecule [5], while reversible oxygen induced fluorescent quenching in poly(9,9-dioctylfluorene) (PF8) neat films was recently demonstrated [6]. A recent breakthrough in the sensitivity enhancement was represented by the demonstration that in neat active films showing optical gain, the Amplified Spontaneous Emission (ASE) and the laser intensity show a stronger relative quenching with respect to the Spontaneous Emission (SE) [7], exploited in efficient explosive vapours detection. This first result was followed by similar demonstration [8] of explosive detection in PF8 and in few evidences of oxygen detection $[9,10]$.
In this letter we demonstrate reversible oxygen induced emission quenching of both the Spontaneous Emission (SE) and the Amplified Spontaneous Emission (ASE) in a PF8 waveguide. We demonstrate that the ASE shows a detection sensitivity up to 6.2 times larger than the SE and a stronger sensitivity decrease as the excitation density increases. The relaxation processes affecting the intensity quenching of both SE and ASE are discussed concluding that the fast ASE rate increase with the excitation density is the process limiting the sensitivity improvement of ASE with respect to SE.

\section{Materials and Methods}

The PF8 film, with a thickness of about $650 \mathrm{~nm}$, was realized by spin coating on a glass substrate of a $310^{-4} \mathrm{M}$ solution in toluene. The sample was pumped by a Nitrogen laser $(337 \mathrm{~nm})$ delivering $3 \mathrm{~ns}$ pulses with a repetition rate of $10 \mathrm{~Hz}$, focused in a $6.6 \mathrm{~mm} \times 100 \mu \mathrm{m}$ rectangular stripe by a cylindrical lens. In order to minimize the photoluminescence (PL) intensity fluctuations due to laser instabilities all the PL spectra were acquired by accumulating the signal due to 10 laser pulses. The PL was collected from the sample edge, dispersed by a TRIAX 320 monochromator with a $150 \mathrm{ll} / \mathrm{mm}$ diffraction grating, and detected by a Peltier cooled Si-Charge Coupled 


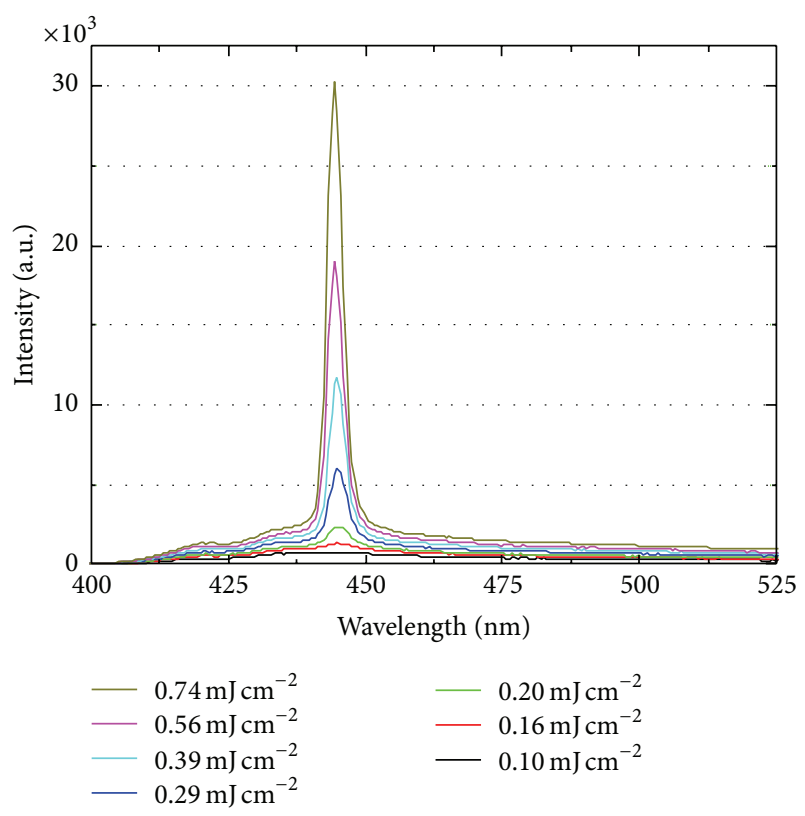

FIGURE 1: Excitation density dependence of the PL spectra measured in vacuum. A clear ASE band is observed in the spectra for excitation density higher than about $120 \mu \mathrm{Jcm}^{-2}$.

Device (CCD). The spectral resolution was about $2 \mathrm{~nm}$. The spectral effect of the oxygen was investigated by comparing the PL spectra measured in air with the ones measured in vacuum (about $10^{-3} \mathrm{mbar}$ ), as a function of the excitation density.

\section{Results and Discussion}

The PL spectra in vacuum (see Figure 1), at low excitation density, show the typical features of the Spontaneous Emission (SE) of the PF8 glassy phase, with a first peak at about $420 \mathrm{~nm}$, followed by the vibronic replica [11]. As the excitation density increases above about $120 \mu \mathrm{Jcm}^{-2}$ a clear band due to Amplified Spontaneous Emission (ASE) is observed at about $445 \mathrm{~nm}$, with an intensity strongly increasing with the excitation density.

When the measurements are performed in air (see Figure 2) no lineshape variations are observed, but the minimum excitation density to observe ASE increases up to about $150 \mu \mathrm{Jcm}^{-2}$. Moreover a clear intensity reduction is observed, at all the investigated excitation densities, both for the SE and for the ASE.

In order to exclude irreversible photodegradation effects due to the strong pumping in air [12], the measurements in vacuum have been repeated after the measurements in air (not shown), leading to the same spectra, intensity, and ASE threshold of the first run of measurements, demonstrating that the observed emission quenching is fully reversible.

Our results are ascribed to oxygen induced reversible quenching, in agreement with previous results [6] on optical oxygen sensing exploiting PF8 SE, and with similar results on ASE quenching $[9,10]$.

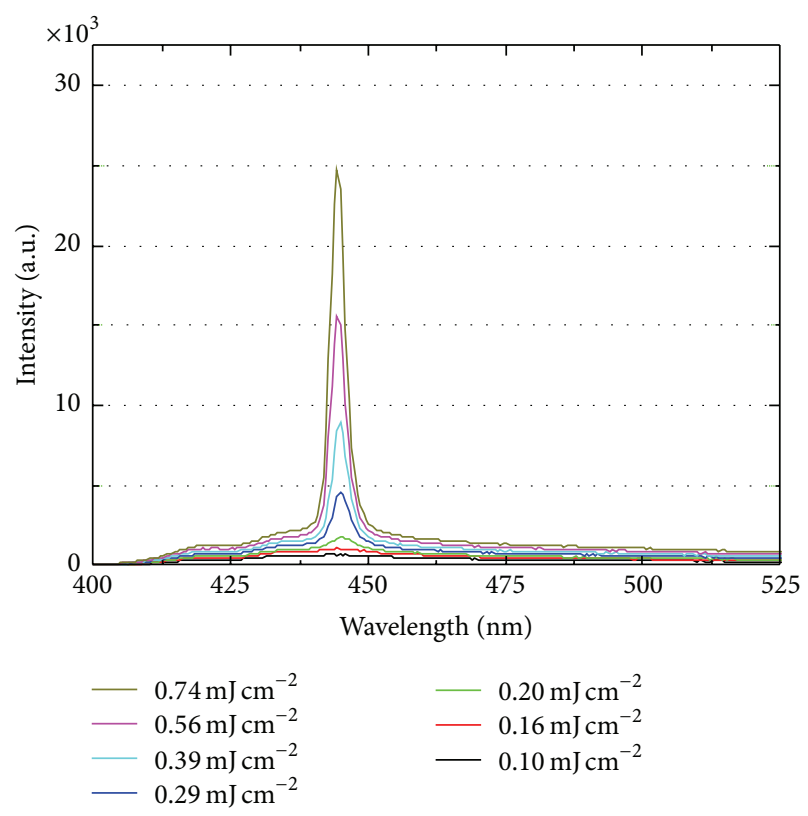

FIGURE 2: Excitation density dependence of the PL spectra measured in air, showing a clear intensity reduction with respect to the spectra in vacuum at the same excitation density.

In order to quantitatively investigate the intensity quenching of SE and ASE and their excitation density dependence we determined the emission intensities in vacuum $\left(I_{\text {vac }}\right)$ and in air $\left(I_{\text {air }}\right)$, by integrating the spectra in the range $410-430 \mathrm{~nm}$ for SE and 441-449 nm for ASE.

The obtained values (see Figure 3 ) show a clear intensity quenching of both signals, larger for the ASE than for the SE band.

The quantitative comparison between the SE and ASE intensity quenching has been based on the determination of the relative intensity quenching defined as

$$
\frac{\Delta I}{I}=\frac{I_{\mathrm{vac}}-I_{\mathrm{air}}}{I_{\mathrm{vac}}}
$$

The main results are (see Figure 4) as follows:

(1) comparable relative quenchings around 10\% are observed in the two spectral bands below the ASE threshold, consistently with a general emission spectra intensity reduction without lineshape variations;

(2) the ASE relative quenching rapidly increases above the ASE threshold, reaches a maximum of $23 \%$ at about $0.2 \mathrm{~mJ} \mathrm{~cm}^{-2}$, and then progressively decreases for higher densities, down to $18 \%$ at $0.74 \mathrm{~mJ} \mathrm{~cm}^{-2}$;

(3) the SE relative quenching progressively decreases with the excitation density, down to about $6 \%$ at $0.74 \mathrm{~mJ} \mathrm{~cm}^{-2}$.

These results clearly show that the detection of the emission intensity of the ASE band allows to increase the oxygen detection sensitivity with respect to the use of SE, up to more than 3 times. It is also interesting to observe that 


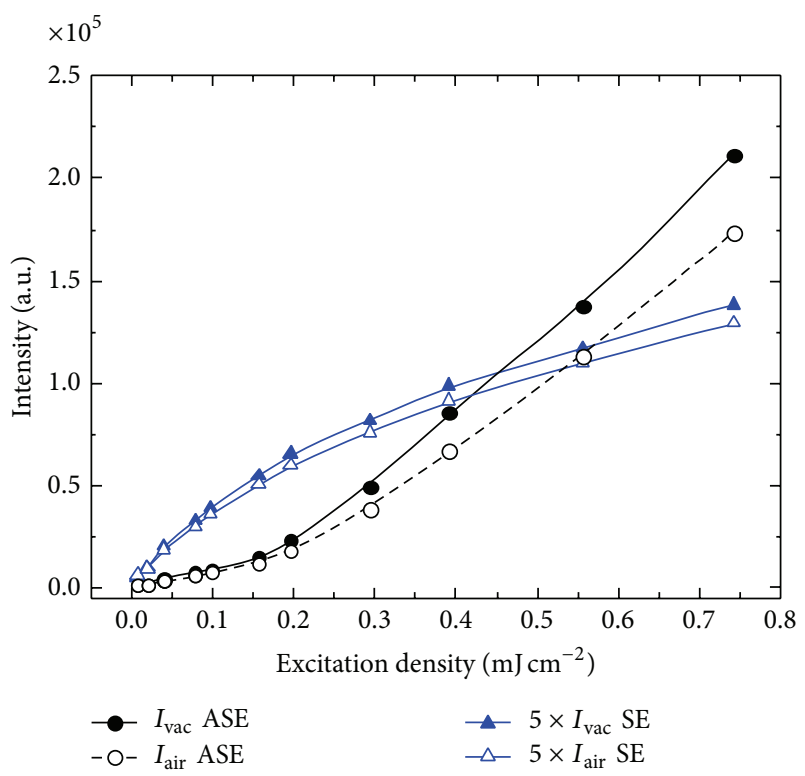

FIGURE 3: Excitation density dependence of the SE and ASE intensity, both in air and in vacuum. The SE intensity has been multiplied by 5 for clarity.

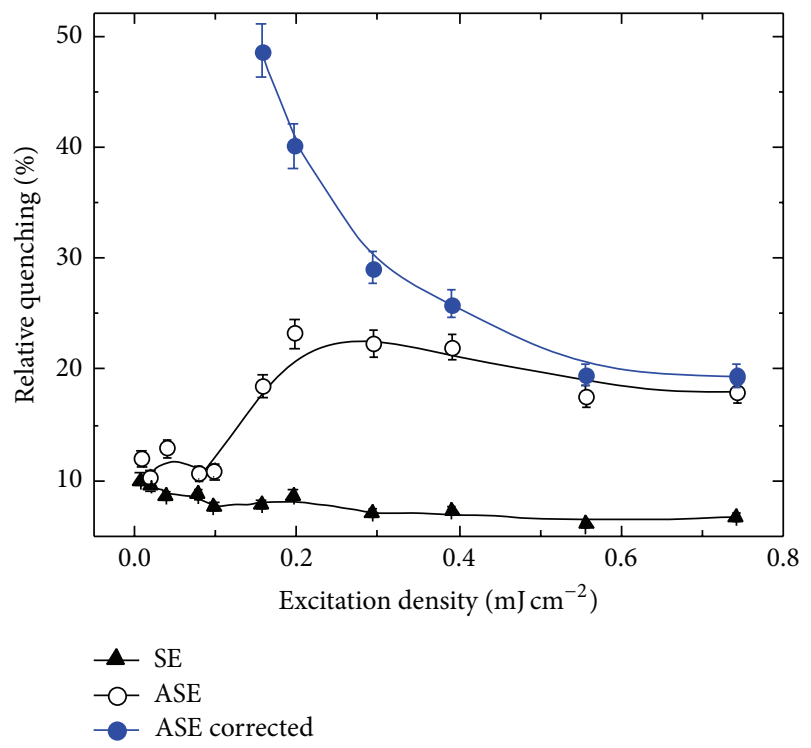

FIGURE 4: Excitation density dependence of the SE, ASE, and corrected ASE relative intensity quenching.

the observed oxygen sensitivity improvement is much higher than the one observed in PF8 based explosive vapours sensors [8] likely suggesting a stronger reversible interaction of PF8 with oxygen with respect to nitroaromatic vapours.

Moreover we observe that the relative quenching of both SE and ASE are clearly dependent on the excitation density. A similar ASE quenching decrease at high excitation density has been to date often observed in similar optical sensing experiments, but it has never been discussed in details [7-10].

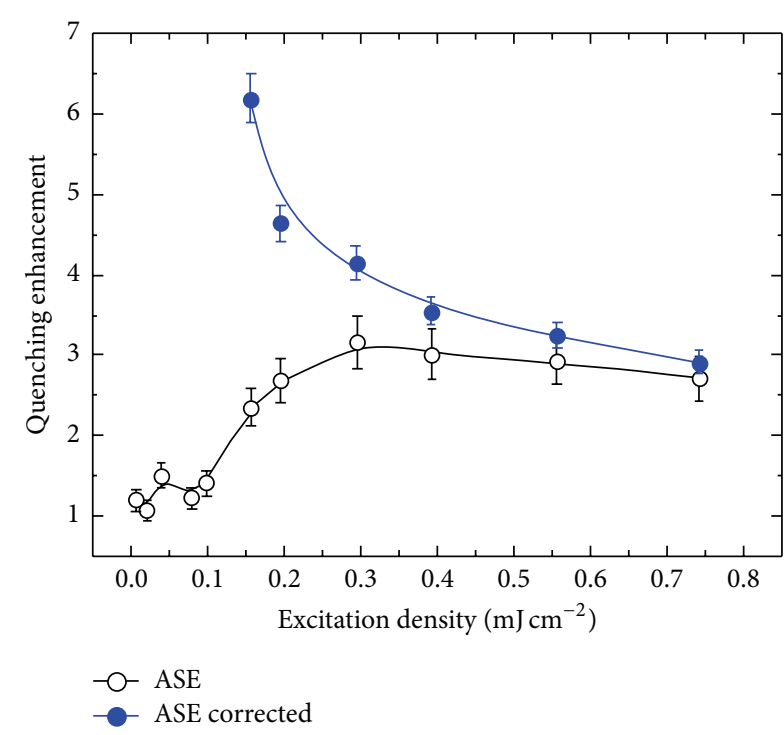

FIGURE 5: Excitation density dependence of the ASE and corrected ASE oxygen sensitivity, relative to the SE one.

As a first step in the understanding of this excitation density dependence we start by observing that the maximum ASE relative quenching is observed just above the ASE threshold, when the relative contribution of the background SE in the ASE intensity detection band is maximum. The detailed ASE quenching investigation then needs the removal of the SE quenching contribution in the ASE spectral region.

When this correction is performed, a maximum corrected ASE relative quenching of about $49 \%$ is observed just above threshold, with a clear progressive decrease down to about $19 \%$ at $0.74 \mathrm{~mJ} \mathrm{~cm}^{-2}$ (see Figure 4).

The oxygen sensitivity improvement obtainable by using the ASE quenching instead of the SE one can be easily estimated by determining the relative ASE/SE quenching (see Figure 5). Our data show that by monitoring the whole sample emission in the ASE spectral region a sensitivity enhancement up to 3.2 times can be obtained at an excitation density about twice the ASE threshold, while by exploiting the corrected ASE quenching the maximum sensitivity is increased up to 6.2 times, but a stronger excitation density dependence is observed, with a progressive decrease down to about 3.1 at the maximum investigated excitation density.

In order to rationalize the excitation density dependence of both the SE and the ASE quenching we observe that the emission quenching depends both on two independent factors, namely, the number of excitons quenched by the PF8-oxygen interaction and the strength of the intensity dependence on the exciton density.

Concerning the strength of the intensity dependence on the exciton density, the SE intensity is directly proportional to the exciton density, while the ASE intensity superlinearly increases with the exciton population (almost exponentially far from saturation [13]). 
Moreover, as the ability of any analyte to quench the active material emission is related to the relative value of the molecule-analyte interaction rate $\left(k_{0}\right)$ with respect to the exciton relaxation rate $(k)$, the fraction of quenched excitons increases with the $k_{0} / k$ ratio.

Considering that in our case the intrinsic exciton relaxation pathways are the Spontaneous Emission and the stimulated emission, the intrinsic exciton relaxation rate is given by $k=k_{\mathrm{SE}}+k_{\mathrm{ASE}}$, where $k_{\mathrm{SE}}$ and $k_{\mathrm{ASE}}$ are the decay rate of SE and ASE, respectively.

Below the ASE threshold $k_{\mathrm{SE}}$ does not depend on the excitation density and $k_{\mathrm{ASE}}=0$; thus the fraction of quenched exciton is independent on the excitation density, resulting in a constant relative intensity quenching, as observed. In this regime the relative intensity quenching directly measures the fraction of quenched excitons, which in our case is then about $10 \%$.

Above the ASE threshold, ASE progressively becomes the main relaxation process due to the progressive increase [14] of $k_{\mathrm{ASE}}$, resulting in a decrease of the fraction of excitons decaying by SE, as evidenced by the sublinear SE increase visible in Figure 3. The $k$ increase also decreases the fraction of quenched exciton, thus proportionally reducing the SE relative quenching, as observed.

The higher ASE sensitivity and its stronger relative decrease when the excitation density increases result from the interplay between the exponential ASE intensity dependence on the exciton density and the excitation density dependence of the fraction of quenched excitons. Just above ASE threshold the fraction of quenched excitons can be expected to be close to just below threshold value (thus still of the order of $10 \%)$, and the exponential ASE intensity dependence on the exciton number leads to a much stronger intensity decrease of the ASE band with respect to the SE one, leading to the observed higher ASE relative quenching with respect to the SE one.

As the excitation density increases the ASE intensity superlinearly increases (see Figure 3), indicating an increase of the strength of the intensity dependence on the excitation density, which should lead to a progressive increase of the ASE relative quenching, which is instead not present. This indicates that the reduction of the fraction of quenched excitons, which is also due to the $k_{\mathrm{ASE}}$ increase, is the dominating factor in determining the sensitivity, resulting in a progressive sensitivity decrease as the excitation density increases.

\section{Conclusions}

In conclusion we observed a reversible oxygen induced quenching of the PF8 SE and ASE, demonstrating that the ASE quenching can allow a higher sensitivity of an oxygen optical sensor. The investigation of the relaxation processes determining the final value of both SE and ASE quenching and of their excitation density dependence suggests that the strong excitation density dependence of $k_{\text {ASE }}$ results in a strong reduction of the exciton quenching efficiency, thus limiting the potentiality of sensitivity improvement of ASE with respect to SE.

This conclusion is expected to be of general validity, as an ASE sensitivity decrease with the excitation density is always reported in similar experiments, and suggests that, rather counterintuitively, the superlinear dependence of the ASE on the exciton density is both the factor determining the sensitivity improvement and the factor limiting the maximum value of the sensitivity.

\section{Conflict of Interests}

The authors declare that there is no conflict of interests regarding the publication of this paper.

\section{References}

[1] Y. Amao, "Probes and polymers for optical sensing of oxygen," Microchimica Acta, vol. 143, no. 1, pp. 1-12, 2003.

[2] K. R. Sridha and J. A. Blanchard, "Electronic conduction in low oxygen partial pressure measurements using an amperometric zirconia oxygen sensor," Sensors and Actuators B, vol. 59, no. 1, pp. 60-67, 1999.

[3] Y. Suzuki, H. Nishide, and E. Tsuchida, "Membranes of the picket fence cobalt porphyrin complexed with poly(vinylimidazole and -pyridine)s: selective optical response to oxygen," Macromolecules, vol. 33, no. 7, pp. 2530-2534, 2000.

[4] G. E. Collins and S. L. Rose-Pehrsson, "Chemiluminescent chemical sensors for oxygen and nitrogen dioxide," Analytical Chemistry, vol. 67, no. 13, pp. 2224-2230, 1995.

[5] P. Douglas and K. Eaton, "Response characteristics of thin film oxygen sensors, Pt and Pd octaethylporphyrins in polymer films," Sensors and Actuators B, vol. 82, no. 2-3, pp. 200-208, 2002.

[6] M. Anni and R. Rella, "Interplay between amplified spontaneous emission, Förster resonant energy transfer, and self-absorption in hybrid poly(9,9-dioctylfluorene)-CdSe/ZnS nanocrystal thin films," Journal of Physical Chemistry B, vol. 114, no. 5, pp. 2086-2090, 2010.

[7] A. Rose, Z. Zhu, C. F. Madigan, T. M. Swager, and V. Bulović, "Sensitivity gains in chemosensing by lasing action in organic polymers," Nature, vol. 434, no. 7035, pp. 876-879, 2005.

[8] Y. Yang, G. A. Turnbull, and I. D. W. Samuel, "Sensitive explosive vapor detection with polyfluorene lasers," Advanced Functional Materials, vol. 20, no. 13, pp. 2093-2097, 2010.

[9] M. Gaal, S. Sax, H. Plank et al., "Directly imprinted surfaceemitting distributed feedback structure polymer sensor laser devices for enhanced oxygen sensitivity," Japanese Journal of Applied Physics, vol. 47, pp. 304-306, 2008.

[10] H.-W. Lin, M.-H. Huang, Y.-H. Chen et al., "Novel oxygen sensor based on terfluorene thin-film and its enhanced sensitivity by stimulated emission," Journal of Materials Chemistry, vol. 22, pp. 13446-13450, 2012.

[11] M. Ariu, M. Sims, M. D. Rahn et al., "Exciton migration in $\beta$ phase poly(9,9-dioctylfluorene)," Physical Review B, vol. 67, no. 19, Article ID 195333, 11 pages, 2003.

[12] M. Anni, "Photodegradation effects on the emission properties of an amplifying poly(9,9-dioctylfluorene) active waveguide operating in air," Journal of Physical Chemistry B, vol. 116, no. 15, pp. 4655-4660, 2012. 
[13] O. Svelto, S. Taccheo, and C. Svelto, "Analysis of amplified spontaneous emission: some corrections to the Linford formula," Optics Communications, vol. 149, no. 4-6, pp. 277-282, 1998.

[14] C. Kallinger, S. Riechel, O. Holderer et al., "Picosecond amplified spontaneous emission bursts from a molecularly doped organic semiconductor," Journal of Applied Physics, vol. 91, no. 10, p. 6367, 2002. 

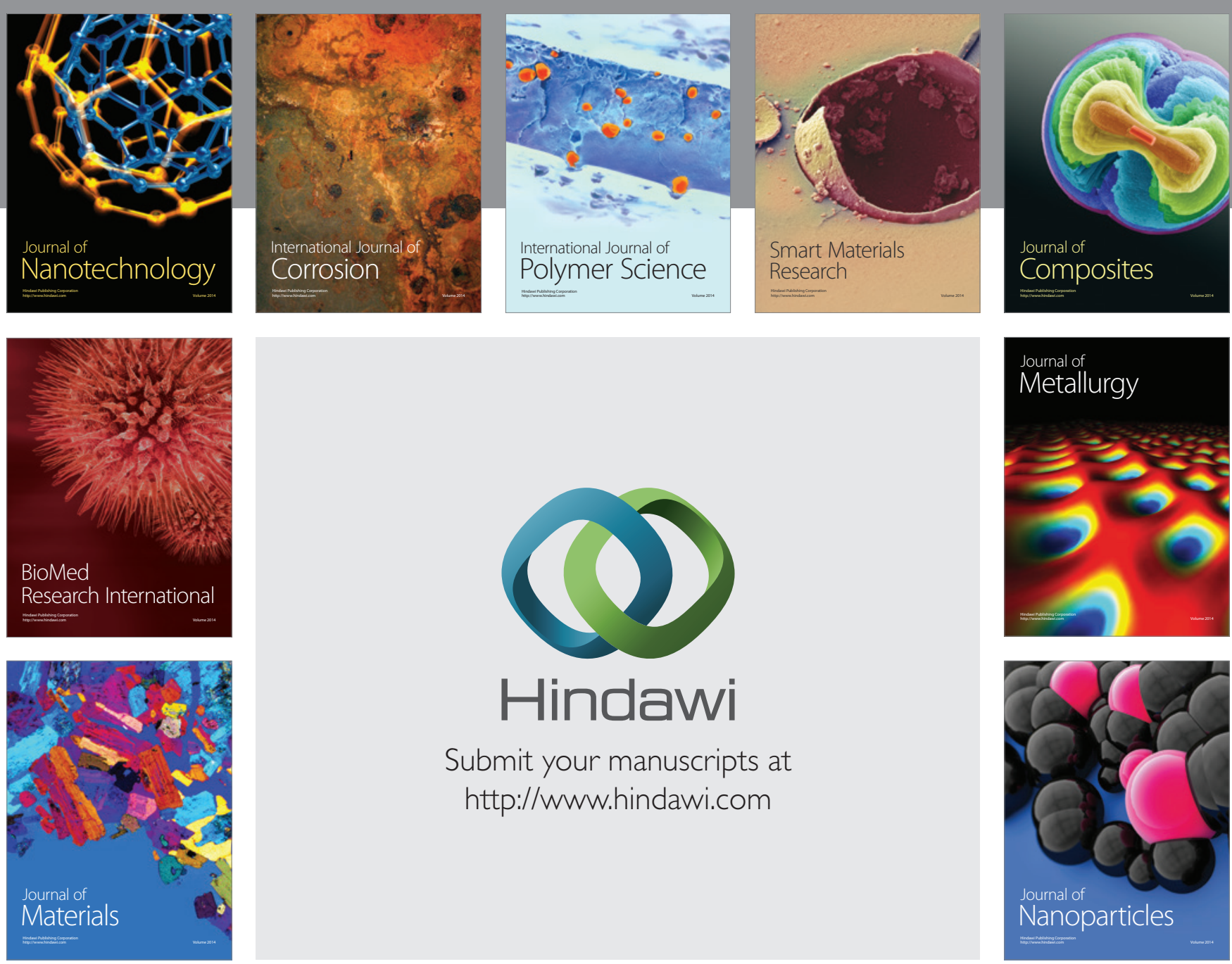

Submit your manuscripts at http://www.hindawi.com
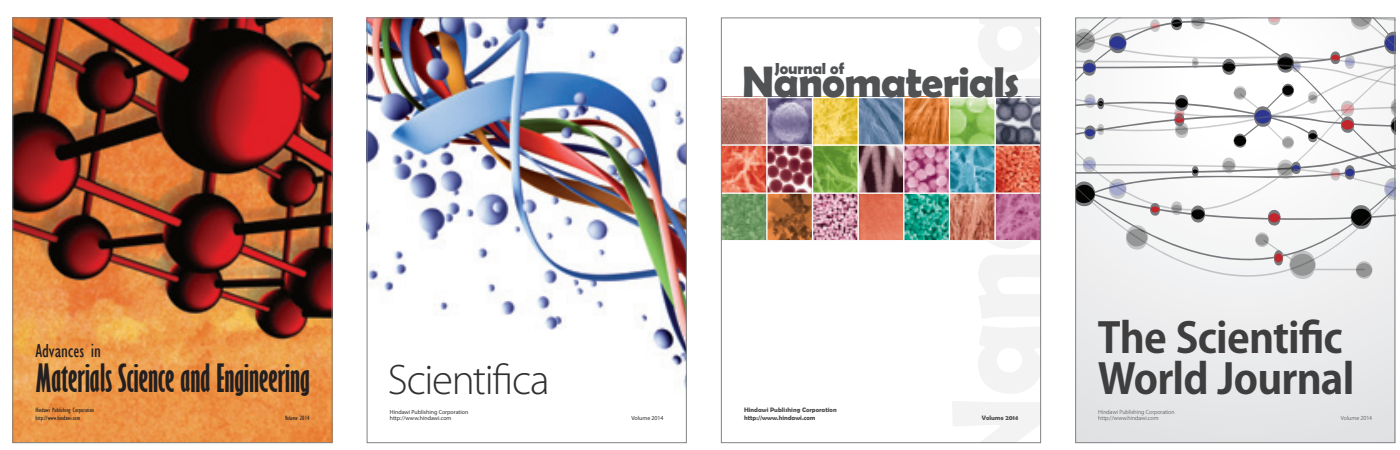

\section{The Scientific World Journal}
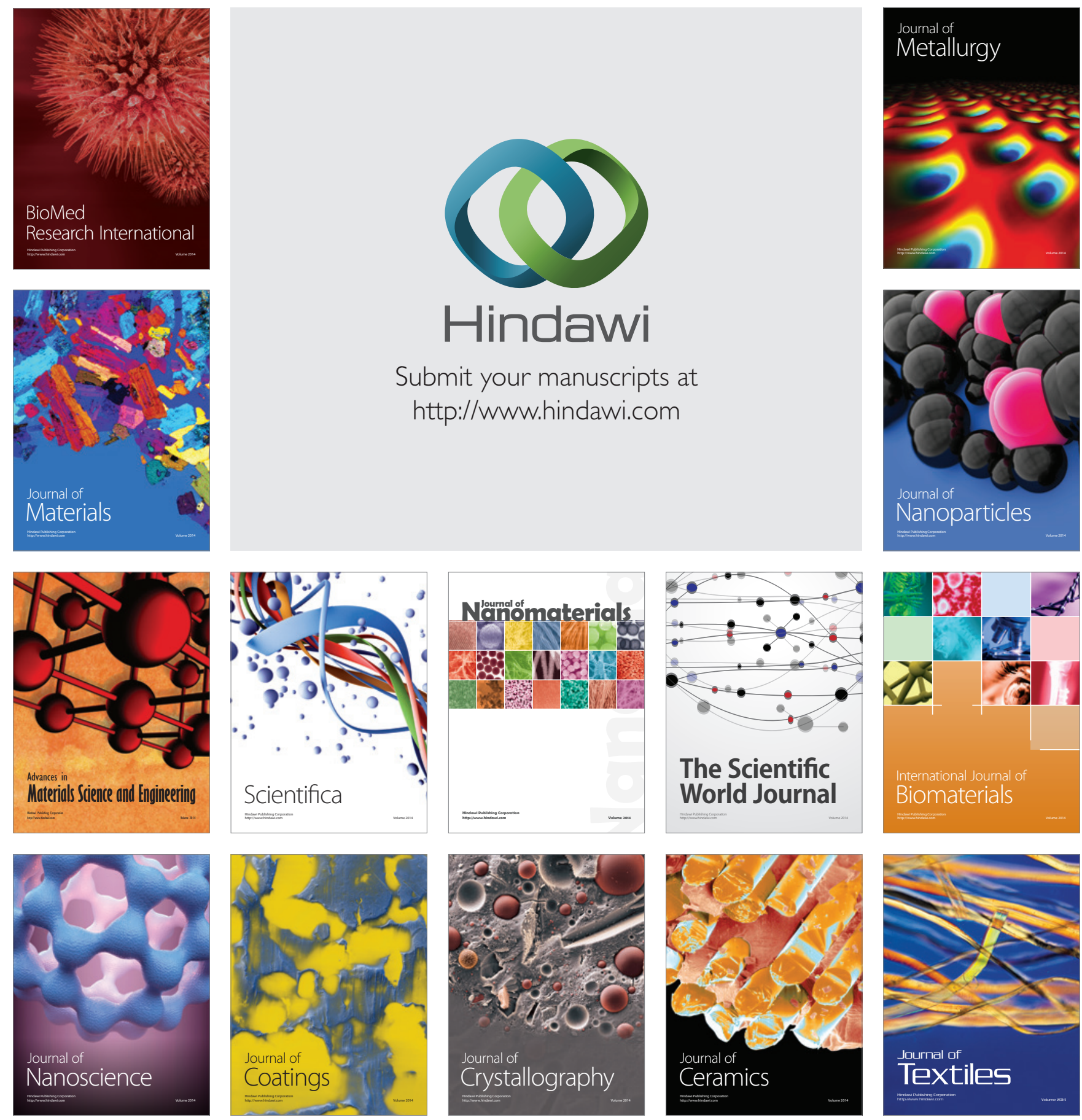